\title{
Stem cells: roadmap to the clinic
}

\author{
George Q. Daley \\ Stem Cell Transplantation Program, Division of Pediatric Hematology/Oncology, Manton Center for Orphan Disease Research, \\ Howard Hughes Medical Institute, Boston, Massachusetts, USA. Children's Hospital Boston and Dana Farber Cancer Institute, Boston, Massachusetts, USA. \\ Division of Hematology, Brigham and Women's Hospital, Boston, Massachusetts, USA. Department of Biological Chemistry and Molecular Pharmacology, \\ Harvard Medical School, Boston, Massachusetts, USA. Harvard Stem Cell Institute, Boston, Massachusetts, USA.
}

\begin{abstract}
Over the last decade, a remarkable number of papers have been published in which the biology of stem cells is introduced with words and phrases such as "promise," "rapid progress," and "future therapies." To separate myth and hype from reality, the articles in this Stem Cells Review series comprise a rich resource on the state of this fast-paced field and provide a balanced perspective on some of the major advances. They recount what the field has achieved over the past decade and where the field is headed. They also highlight the challenges to be faced in translating what is indeed highly promising science into proven therapies that will regenerate and repair diseased tissues.
\end{abstract}

\section{Stem cell biology as a scientific discipline}

Stem cell biology has emerged from the intellectual traditions of cell and developmental biology to occupy an ever more prominent role in modern biology and medicine. While developmental biology focuses on how the embryo is made, stem cell biology addresses how tissues and organs are formed and maintained throughout life. Given the common themes of stem cell function, cell and molecular biologists from previously disparate areas of medicine - including neuroscience, hematology, cardiology, gastroenterology, and dermatology - have organized themselves into new intellectual communities and institutional structures. With the genetic code in hand, deciphering information in the genome is increasingly the provenance of stem cell biology, a discipline that endeavors to define the connections between epigenetic regulation and cell fate. The scientific mission of stem cell biology is to reveal how genetic information is translated into tissue formation and organogenesis.

Stem cell biology also encompasses applications for treating diseases of tissue malformation, degeneration, trauma, and genetic deficiency, and efforts to translate scientific insights into new therapies is gaining momentum. Cell-based assays that employ differentiated products of stem cells represent a novel strategy for chemical biology and small molecule drug discovery and indeed promise to provide drugs that enhance natural repair and regeneration. Moreover, stem cells offer the promise that delivering cells will restore function to diseased tissues. This seductive combination of exciting fundamental scientific questions and opportunities to contribute to human health have made stem cell biology a magnet for talented young students and changed the careers of even seasoned investigators. It is hoped that the articles in this Review series, which cover some of the most compelling opportunities in stem cell biology, comprise a rich resource for all these individuals as well as those wishing to learn more about this exciting field.

\section{Developmental potency and the intrinsic nature of stem cells}

Fundamental to an understanding of the function and potential clinical applications of different types of stem cells is the concept of developmental potency, which refers to the range of possible fates open to cells during differentiation. It is generally the case

Conflict of interest: The author is a member of the scientific advisory boards and holds equity in MPM Capital, iPierian Inc., Epizyme Inc., and Solasia Pharma K.K. Citation for this article: J. Clin. Invest. 120:8-10 (2010). doi:10.1172/JCI41801. that cellular potency is progressively restricted as development proceeds from a fertilized egg to the adult. Stem cells are an exception to this rule in that they retain, to varying extents, the potential for multi-lineage differentiation; therein rests one of the most significant properties of this distinct class of cells. One could argue that fertilized eggs represent the pinnacle of the cellular hierarchy of developmental potency. They are totipotent by virtue of their ability to orchestrate the formation of an entire organism. However, unlike stem cells, fertilized eggs don't self-renew by simple cell division. ES cells derived from the early blastocyst are therefore the most potent of stem cells, capable of unlimited growth in tissue culture and able to give rise to all cell types of the developing soma (but typically not the extra-embryonic structures such as the placenta). When murine ES cells are returned to the blastocyst by microinjection, they chimerize all tissues of the developing animal but not the placenta (1), which reflects their developmental exclusion from the trophectoderm lineage. As such, ES cells lack totipotency and are considered pluripotent. Although for years human ES (hES) cells were considered the developmental equivalents of mouse ES cells, it now appears that hES cells are more like murine epiblast-derived stem cells (Epi-SCs; one of the earliest cell lineages of the embryo that gives rise to the fetus itself) $(2,3)$ and thus derive from a slightly later stage of embryonic development. The developmental potency of hES cells has not, of course, been tested by chimerizing human embryos, but if they behave like mouse Epi-SCs, they would not chimerize extensively, although they do show substantial pluripotency in vitro. In immunocompromised mice, hES cells produce teratomas, encapsulated tumors consisting of disorganized masses of differentiated tissues from all three embryonic germ layers, which is the most stringent test of pluripotency currently in use today (4). Because of their pluripotency, ES cells represent important tools for analyzing the relationships between gene function and cell and tissue formation. Through directed differentiation into specific tissues, ES cells may also provide a source of cells for transplantation therapy, although defining the specific cell types and the routes of transplantation and engraftment, and overcoming the immune barrier, represent major challenges for realizing the potential of cell-based therapy.

In contrast to the pluripotent cells of the early embryo and some derivatives of the germ lineage, somatic cells of the developing embryo assume increasing degrees of fate restriction as they specialize into specific tissue lineages. Highly proliferative tissues that turn over rapidly throughout adulthood, replacing their mass in a 
matter of days - for example the blood, skin, and gut epithelia regenerate from a pool of somatic stem cells (also known as adult stem cells, which invites confusion because they can be extracted from newborns and adolescents and therefore not strictly adult sources). Somatic stem cells actively replenish themselves through self-renewal and regenerate the several cell types that comprise their respective tissues. Somatic stem cells are thus deemed multipotent to reflect their multiple yet tissue-restricted range of fates.

In contrast to highly proliferative tissues sustained by self-renewing, multipotent somatic stem cells, tissues that turn over more slowly appear to follow distinct mechanisms of tissue regeneration and repair that might not involve true stem cells. The anatomically complex tissues of the brain, lung, heart, and kidney manifest limited regenerative potential. Indeed, given the fact that many tissues are highly stable and undergo scarring rather than regeneration in response to cellular injury, it is probable that somatic stem cells will not be available for repair of these tissues. The role of resident stem cells in the mature adult heart, and their facultative contributions to tissue repair and regeneration (as opposed to their role in steady-state maintenance of tissue integrity), remains a controversial subject. Similarly, there is controversy about the nature of repair or regeneration of pancreatic islets, especially over the nature of $\beta$ cell replacement for the intended purpose of treating diabetes. In response to partial hepatectomy, regeneration of the hepatic parenchyma occurs by division of pre-existing hepatocytes (5), a mechanism distinct from replenishment from a stem cell pool. However, following organ injury, hepatocytes can derive de novo from oval cells, progenitors claimed to be capable of forming ductal cells as well (5). Oval cells thus appear to be facultative sources of cellular repair, as they are relatively dormant until recruited into the cell cycle. Similarly, muscle stem/satellite cells divide and can repopulate injured muscle by generating differentiated myofiber de novo (6). The functions of both highly proliferative and facultative somatic stem cells are being actively investigated. Drugs that act on endogenous stem cells are under development, and transplantation strategies involving somatic stem cells (chiefly mesenchymal stem cells and hematopoietic stem cells) are being actively developed. Only studies that use hematopoietic stem cells have become established as the standard of care for malignant and genetic bone marrow disorders. All other applications remain highly experimental at this time.

\section{Engineering pluripotency}

Perhaps the most transformative contribution to the field of stem cell biology in the last decade is the engineering of pluripotency into somatic cells by the ectopic expression of transcription factors linked to pluripotency. Culling 24 genes from the large number expressed in ES cells, Takahashi and Yamanaka created a minilibrary of candidates believed important to pluripotency, then transduced mouse fibroblasts and selected for cells reminiscent of ES cells (7). Indeed, such "reprogrammed" cells appeared as ES cell-like colonies. By repeating the experiment 24 times, each iteration lacking one gene candidate, they determined pools that lacked reprogramming activity, which pointed directly to the essential factors. Ultimately, the transcription factors octamer 3/4 (Oct4), SRY box-containing gene 2 (Sox2), Kruppel-like factor 4 (Klf4), and Myc proved sufficient to induce pluripotency in somatic fibroblasts (7). The resulting induced pluripotent stem cells (iPS cells) represent the functional equivalents of ES cells in terms of multi-lineage differentiation in vitro, teratoma formation, germline transmission, and even the ability to create an entire embryo from iPS cells injected into tetraploid blastocysts (which alone cannot support somatic development). A little more than a year after the original demonstration of direct reprogramming, several groups had refined the method in mice and extended reprogramming to human cells (8-12). Reprogramming now represents a powerful new technique for engineering the fate of cells for both scientific and medical ends.

Factor-based reprogramming enables one of the long-standing ambitions of stem cell biology: the ability to generate pluripotent stem cells from specific patients (13). Figuratively, direct reprogramming can move a patient's disease into the Petri dish. For diseases with a clear cellular phenotype - and there are many, for example immunodeficiency, sickle cell anemia, and polycythemia, all of which involve blood lineages, and Parkinson disease and amyotrophic lateral sclerosis, which entail loss of specific types of neurons - the act of differentiation can recapitulate the formation of vulnerable or aberrant tissue populations, allowing the pathological to be discerned from the normal. The metaphor of the flight data recorder has been used to explain how creating diseased tissues from patient-derived iPS cells allows one to replay the disease process while listening for clues to the destruction ahead. If the cell-intrinsic defect is profound enough to be observed in vitro, there is the prospect that screening chemical libraries might identify "hits" that restore normal function and thus could serve as the basis for drug development. Alternatively, given that iPS cells represent a patient's own genetic make-up, any tissue derived from the line would necessarily be histocompatible, allowing for rejection-proof cell transplantation. Whereas studies of ES cells afford great opportunities to ask generic questions about cellular differentiation, iPS cells afford the opportunity to model specific diseases in vitro and the prospects for autologous cell therapy. Undoubtedly, significant challenges must be overcome before that promise is realized.

\section{When might we expect clinical success?}

Given the enormous attention being paid to the field of stem cell biology, a frequent question is when we might witness successful clinical application(s). If history is any guide, the development of new medical modalities takes many years to find full and fruitful application. The recombinant DNA methods introduced in the 1970s led quickly to the development and FDA approval of human insulin in 1982. However, it took until the 1990s - some twenty years later - before protein-based pharmaceuticals became billiondollar blockbusters for the biotechnology industry. Monoclonal antibodies, first described in 1975, became approved therapeutic products with the introduction of OKT3, which combats organ transplant rejection, in 1986, but they became widely successful in the treatment of cancer only more recently. Thus, if history is our guide, it takes some two decades for a new biotechnology advance to translate into widely successful clinical therapies. Given that hES cells were first described in 1998 (14), and human iPS cells only in late $2007(9,10,12)$, we might anticipate some $10-15$ years before effective products are developed, thereby launching the era of regenerative medicine.

\section{The Review series}

The articles in this Stem Cells Review series tackle several of the most interesting, promising, and vexing topics in stem cell biology and regenerative medicine, and all are written by acknowledged leaders in their respective fields. Giulio Cossu and colleagues 
review the remarkable progress in defining progenitors responsible for repair and regeneration of skeletal muscle (15). Understanding muscle repair will be essential for treating conditions as diverse as muscular dystrophy and the sarcopenia of aging. Kenneth Chien, Alexander Yi, and Oliver Wernet analyze the ontogeny of cardiomyocyte development and recount how specific cardiac progenitors arise (16). Whether stem cells exist in adult hearts and can be coaxed to enhance myocardial repair and regeneration remains a contentious debate, but there is no disagreement that insights into myocardial development offered by the study of cardiomyocyte differentiation in vitro does indeed give hope for addressing the large unmet need in heart failure and cardiovascular disease. Cell therapy has been attempted in Parkinson disease for several decades, with mixed results that are celebrated by some as successes warranting further investigation, while being critiqued by others. Olle Lindvall, a leader in cellular transplantation for Parkinson disease, and his colleague Zaal Kokaia provide a historical and a futuristic accounting of the prospects for cell therapy in Parkinson disease and other neurodegenerative conditions (17). Cancer stem cells, particularly those relevant to melanoma, are described by Markus Frank, Tobias Schatton, and Natasha Frank (18). Insights into self-renewal pathways and the discovery of unique markers that discriminate cancer stem cells from the bulk population of tumor cells offer hope for improved cancer therapeutics and may prove to be one of the areas where stem cell insights translate most quickly to the clinic. The exciting opportunities offered by iPS cells are reviewed by Kevin Eggan and Evangelos Kiskinis (19), while the critical challenges of defining the requisite microenvironmental conditions to promote directed stem cell differentiation are discussed by Peter Zandstra and Raheem Peerani (20). Finally, bioethicist Insoo Hyun reviews the ethical debate that has kept stem cell biology in the limelight for so long (21). Although the edge of the hES cell debate has been dulled by the advent of iPS cells, ethical challenges remain, especially "stem cell tourism," which thrives as clinics around the world rush to market unproven therapies to vulnerable patients. Hyun and Lindvall co-chaired the special task force that produced the Guidelines for the Clinical Translation of Stem Cells on behalf of the International Society for Stem Cell Research (22), which represents the true "roadmap" for responsible translation of stem cell science. It is thus fitting that Hyun gets the last word in the series.

\section{Acknowledgments}

The stem cell and cancer studies in the author's laboratory have been supported by the NIH, the Leukemia and Lymphoma Society, the Burroughs Wellcome Fund, the Manton Foundation, the Roche Foundation for Anemia Research, Alex's Lemonade Stand, the Thomas Anthony Pappas Charitable Foundation, Harvard Catalyst, the Howard Hughes Medical Institute, and private funds donated to the Harvard Stem Cell Institute and Children's Hospital Boston.

Address correspondence to: George Q. Daley, Children's Hospital Boston, Karp Family Research Building 7214, 300 Longwood Avenue, Boston, Massachusetts 02115, USA. Phone: (617) 919-2013; Fax: (617) 730-0222; E-mail: george.daley@childrens.harvard.edu.
1. Lu CW, Yabuuchi A, Chen L, Viswanathan S Kim K, Daley GQ. Ras-MAPK signaling promotes trophectoderm formation from embryonic stem cells and mouse embryos. Nat Genet. 2008;40(7):921-926.

2. Brons IG, et al. Derivation of pluripotent epiblast stem cells from mammalian embryos. Nature. 2007;448(7150):191-195.

3. Tesar PJ, et al. New cell lines from mouse epiblast share defining features with human embryonic stem cells. Nature. 2007;448(7150):196-199.

4. Lensch MW, Schlaeger TM, Zon LI, Daley GQ. Teratoma formation assays with human embryonic stem cells: a rationale for one type of human-animal chimera. Cell Stem Cell. 2007;1(3):253-258.

5. Zaret KS, Grompe M. Generation and regeneration of cells of the liver and pancreas. Science. 2008; 322(5907):1490-1494.

6. Cerletti M, Shadrach JL, Jurga S, Sherwood R, Wagers AJ. Regulation and function of skeletal muscle stem cells. Cold Spring Harb Symp Quant Biol. 2008;73:317-322

7. Takahashi K, Yamanaka S. Induction of pluripotent stem cells from mouse embryonic and adult fibroblast cultures by defined factors. Cell. 2006; 126(4):663-676.

8. Maherali $\mathrm{N}$, et al. Directly reprogrammed fibroblasts show global epigenetic remodeling and widespread tissue contribution. Cell Stem Cell. 2007;1(1):55-70

9. Park IH, et al Reprogramming of human somatic cells to pluripotency with defined factors. Nature. 2008;451(7175):141-146.

10. Takahashi $\mathrm{K}$, et al. Induction of pluripotent stem cells from adult human fibroblasts by defined factors. Cell. 2007;131(5):861-872.

11. Wernig $M$, et al. In vitro reprogramming of fibroblasts into a pluripotent ES-cell-like state. Nature. 2007;448(7151):318-324.

12. Yu J, et al. Induced pluripotent stem cell lines derived from human somatic cells. Science. 2007; 318(5858):1917-1920.

13. Park IH, et al. Disease-specific induced pluripotent stem cells. Cell. 2008;134(5):877-886.

14. Thomson JA, et al. Embryonic stem cell lines derived from human blastocysts. Science. 1998; 282(5391):1145-1147.

15. Tedesco FS, Dellavalle A, Diaz-Manera J, Messina G,
Cossu G. Repairing skeletal muscle: regenerative potential of skeletal muscle stem cells. J Clin Invest. 2010;120(1):11-19.

16. Yi BA, Wernet O, Chien KR. Pregenerative medicine: developmental paradigms in the biology of cardiovascular regeneration. J Clin Invest. 2010; 120(1):20-28.

17. Lindvall O, Kokaia Z. Stem cells in human neurodegenerative disorders - time for clinical translation? J Clin Invest. 2010;120(1):29-40.

18. Frank NY, Schatton T, Frank MH. The therapeutic promise of the cancer stem cell concept. J Clin Invest. 2010;120(1):41-50.

19. Kiskinis E, Eggan K. Progress toward the clinical application of patient-specific pluripotent stem cells. J Clin Invest. 2010;120(1):51-59.

20. Peerani R, Zandstra PW. Enabling stem cell therapies through synthetic stem cell-niche engineering. J Clin Invest. 2010;120(1):60-70.

21. Hyun I. The bioethics of stem cell research and therapy. J Clin Invest. 2010;120(1):71-75.

22. Hyun I, et al. New ISSCR guidelines underscore major principles for responsible translational stem cell research. Cell Stem Cell. 2008;3(6):607-609. 